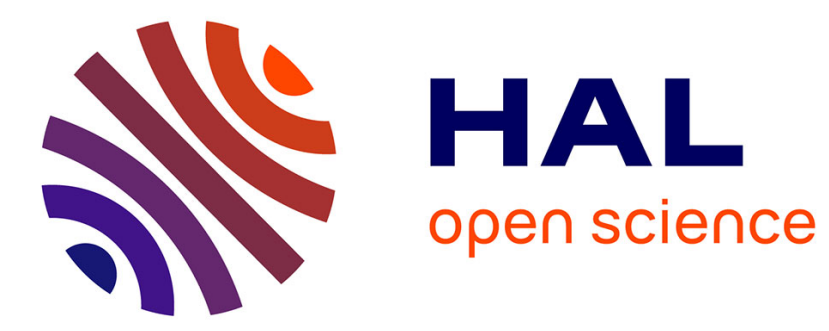

\title{
Construction of a control for the cubic semilinear heat equation
}

\author{
Thi Minh Nhat Vo
}

\section{To cite this version:}

Thi Minh Nhat Vo. Construction of a control for the cubic semilinear heat equation. Vietnam Journal of Mathematics, 2015, 10.1007/s10013-015-0171-x . hal-01225203

\section{HAL Id: hal-01225203 \\ https://hal.science/hal-01225203}

Submitted on 9 Nov 2015

HAL is a multi-disciplinary open access archive for the deposit and dissemination of scientific research documents, whether they are published or not. The documents may come from teaching and research institutions in France or abroad, or from public or private research centers.
L'archive ouverte pluridisciplinaire HAL, est destinée au dépôt et à la diffusion de documents scientifiques de niveau recherche, publiés ou non, émanant des établissements d'enseignement et de recherche français ou étrangers, des laboratoires publics ou privés. 


\title{
Construction of a control for the cubic semilinear heat equation
}

\author{
Thi Minh Nhat VO *
}

\begin{abstract}
In this article, we consider the null controllability problem for the cubic semilinear heat equation in bounded domains $\Omega$ of $\mathbb{R}^{n}, n \geq 3$ with Dirichlet boundary conditions for small initial data. A constructive way to compute a control function acting on any nonempty open subset $\omega$ of $\Omega$ is given such that the corresponding solution of the cubic semilinear heat equation can be driven to zero at a given final time $T$. Furthermore, we provide a quantitative estimate for the smallness of the size of the initial data with respect to $T$ that ensures the null controllability property.
\end{abstract}

Keywords. null controllability, cubic semilinear heat equation, linear heat equation.

2010 Mathematics Subject Classification. Primary: 35K58; Secondary: 93 B05.

\section{Introduction and main result}

Many systems in physics, mechanics, or more recently in biology or medical sciences are described by Partial Differential Equations (PDEs). It is necessary to control the characteristic variables, such as the speed of a fluid or the temperature of a device..., to guarantee that a bridge will not collapse or the temperature is at the desired level for example...In the specific words, given a time interval $(0, T)$, an initial state and a final one, we have to find a suitable control such that the solution matches both the initial state at time $t=0$ and the final one at time $t=T$. Let $\Omega$ be a bounded connected open set in $\mathbb{R}^{n}(n \geq 3)$ with a boundary $\partial \Omega$ of class $C^{2} ; \omega$ be a nonempty open subset in $\Omega$.

*Université Paris 13, Sorbonne Paris Cité, LAGA, CNRS UMR 7539, Institut Galilée, 99, Avenue J.-B. Clément F-93430 Villetaneuse Cedex, France; Université d'Orléans, Laboratoire MAPMO, CNRS UMR 7349, Fédération Denis Poisson, FR CNRS 2964, Bâtiment de Mathématiques, B.P. 6759, 45067 Orléans Cedex 2, France; Ho Chi Minh City University of Natural Science, Ho Chi Minh City, Viet Nam. (vtmnhat@hcmpreuedu.vn). This work was written while the author was visiting the University of Orleans (France). She thanks the MAPMO department of mathematics of the University of Orleans. The author also wishes to acknowledge Region Centre for its financial support. 
Consider the cubic semilinear heat equation complemented with initial and Dirichlet boundary conditions, which has the following form:

$$
\begin{cases}\partial_{t} y-\Delta y+\gamma y^{3}=\left.1\right|_{\omega} u & \text { in } \Omega \times(0, T), \\ y=0 & \text { on } \partial \Omega \times(0, T), \\ y(\cdot, 0)=y^{0} & \text { in } \Omega\end{cases}
$$

where $\gamma \in\{1,-1\}$. Well-posedness property and blow-up phenomena for the cubic semilinear heat equation are now well-known results (see e.g. [2] and [4]). It will be said that (1) is null controllable at time $\mathrm{T}$ if there exists a control function $u$ such that the corresponding initial boundary problem possesses a solution $y$ which is null at final time $T$. The basic discussion of this article is how to construct a control function that leads to the null controllability property of system (1).

Our main result is the following:

Theorem 1 There exists a constant $G>1$ such that for any $T>0$, any $y^{0} \in H_{0}^{1}(\Omega)$ satisfying

$$
\left\|y^{0}\right\|_{H_{0}^{1}(\Omega)}^{2} \leq \max _{[0 ; T]} \frac{1}{G(1+t)^{2} \sqrt{t} e^{\frac{G}{t}}}
$$

there exists a control function $u \in L^{2}(\omega \times(0, T))$ such that the solution of (1) satisfies $y(\cdot, T)=0$. Furthermore, the control can be computed explicitly and the construction of the control is given below.

\section{Remarks}

$1 /$ Theorem 1 ensures the local null controllability of (1) for any control set $\omega$, any small enough initial data $y^{0} \in H_{0}^{1}(\Omega)$, at any time $T$. It is well-known that the system (1) without control function blows up in finite time for the case $\gamma=-1$. But thanks to an appropriate control function, Theorem 1 affirms that the blow-up phenomena can be prevented for very specific initial data. This issue (i.e., the null controllability for semilinear heat equations) has been extensively studied (see e.g. [1], [7], [6], [5] and the references therein). Obviously, the result is not new from the point of view of null controllability, but the method completely differs from others.

2/ An important achievement of our result is that we can construct the control function. An outline of the construction is described as follows: firstly, we remind the construction of the control for the linear heat equation with an estimate of the cost (see e.g [13] or [5]); secondly, from the previous result, we do similarly when adding an outside force using the method of Y. Liu, T. Takahashi and M. Tucsnak in [9]. The solution will be forced to be null at time $T$ by adding an exponential weight function; lastly, thanks to an appropriate iterative fixed point process and linearization by replacing the outside force by cubic function, the desired control is constructed, but the result is only local, i.e. the initial condition must be small enough. The precise construction of the control function is found in the proof of this Theorem 1. 
3/ Another main achievement of our result is to give a quantitative estimate for the smallness of the size of the initial condition with respect to the control time $T$. The upper bound of initial data is a function with respect to the final control time $T$, which obviously increases to a certain value and then keeps to be a constant until $T$ tends to $\infty$.

\section{Background}

We now review the achievements of controllability for the heat equations which has been intensively studied in the past. Consider the heat equation in the following form:

$$
\begin{cases}\partial_{t} y-\Delta y+c(t, x) y+f(t, x, y)=\left.1\right|_{\omega} u+g & \text { in } \Omega \times(0, T) \\ y=0 & \text { on } \partial \Omega \times(0, T) \\ y(\cdot, 0)=y^{0} & \text { in } \Omega\end{cases}
$$

For linear case with $f \equiv 0,(2)$ is null controllable with no restriction on $y^{0}, T$ and $\omega$, which means the global null controllability holds. There are at least two ways to approach such result. The first one is due to G. Lebeau and L. Robbiano [8], who connect null controllability to an interpolation estimate for elliptic system. The second one is due to A. Fursikov and O. Imanuvilov [7], and is based on a global Carleman inequality which is an estimate with an exponential weight function and on a minimization technique to construct the control function. For nonlinear case, A. Fursikov and O. Imanuvilov [7] also give us the proof of global null controllability when $f(t, x, s)$ satisfies the global Lipschitz condition in $s$ variable with $f(t, x, 0) \equiv 0$ by means of Schauder's fixed point theorem, and assert the local null controllability when $f(t, x, s)$ satisfies the superlinear growth condition in $s$ by means of the implicit function theorem. In [7], A. Fursikov and O. Imanuvilov point out that null controllability works in case the initial data is small enough but without an explicit formula. In addition, S. Anita and D. Tataru [1] improve the result of A. Fursikov and O. Imanuvilov by providing sharp estimates for the controllability time in terms of the size of the initial data. A little bit different from this document, in [6], E. Fernández-Cara and E. Zuazua establish the first result in the literature on the null controllability of blowing-up semilinear heat equation. In detail, they prove that the system is null-controllable at any time provided a globally defined and bounded trajectory exists and the nonlinear term $f(x, t, s)$ is such that $|f(s)|$ grows slower than $|s| \log ^{\frac{3}{2}}(1+|s|)$ as $|s| \rightarrow \infty$. Furthermore, they observe that it is not possible to obtain a global controllability result for a cubic nonlinear term. More recently, the controllability of a parabolic system with a cubic coupling term has been studied by J-M. Coron, S. Guerrero and L. Rosier in [3].

Another interesting problem is to study the case where the blow-up phenomena will not occur, for example when $\gamma=1$. Our method gives the following result: 
Corollary 1 There exists a constant $G>1$ such that for any $T>0$, any $y^{0} \in L^{2}(\Omega)$ satisfying

$$
\left\|y^{0}\right\|_{L^{2}(\Omega)}^{2} \leq \max _{[0 ; T]} \frac{T}{G(1+t)^{2} \sqrt{t} e^{\frac{G}{t}}},
$$

there exists a control function $u \in L^{2}(\omega \times(0, T))$ such that the solution of (1) with $\gamma=1$ satisfies $y(\cdot, T)=0$.

The article is organized as follows. In section 2 , we deal with the linear heat equation. The construction of the control for the linear heat equation with outside force is described there. In section 3, we apply this construction with a fixed point argument in order to prove the main results: Theorem 1; Corollary 1.

\section{Linear cases}

In this section, we survey the null controllability properties for the linear heat equation.

\subsection{Basic linear case}

Now we recall the results about the null controllability and observability for linear heat equation.

Theorem 2 For any $T>0$ and any $z_{0} \in L^{2}(\Omega)$, there exists a control function $u \in$ $L^{2}(\omega \times(0, T))$ such that the solution $z$ of

$$
\begin{cases}\partial_{t} z-\Delta z=\left.1\right|_{\omega} u & \text { in } \Omega \times(0, T) \\ z=0 & \text { on } \partial \Omega(0, T) \\ z(\cdot, 0)=z_{0} & \text { in } \Omega\end{cases}
$$

satisfies $z(\cdot, T)=0$ in $\Omega$. Furthermore, $u$ can be chosen such that the following estimate holds:

$$
\|u\|_{L^{2}(\omega \times(0, T))} \leq C e^{\frac{C}{T}}\left\|z_{0}\right\|_{L^{2}(\Omega)} \quad \text { for some positive constant } C=C(\Omega, \omega) .
$$

The positive constant $C$ is given in the following equivalent theorem (observability estimate for the heat equation). 
Theorem 3 There exists a constant $C>0$ such that, for any $T>0$, for each $\phi_{T} \in$ $L^{2}(\Omega)$, the associated solution of the system

$$
\begin{cases}\partial_{t} \phi+\Delta \phi=0 & \text { in } \quad \Omega \times(0, T), \\ \phi=0 & \text { on } \quad \partial \Omega \times(0, T) \\ \phi(\cdot, T)=\phi_{T} & \text { in } \Omega\end{cases}
$$

satisfies

$$
\|\phi(\cdot, 0)\|_{L^{2}(\Omega)} \leq C e^{\frac{C}{T}}\|\phi\|_{L^{2}(\omega \times(0, T))} .
$$

The two above results are quite an old subject which started at least from the works of [8] and [7]. Many improvements are given in [13], [6], [5], [12], [10], [11]. We turn now to study the null controllability problem for linear case, but with an outside force.

\subsection{Linear case with the outside force}

Consider the linear heat equation with the outside force, which has the following form:

$$
\begin{cases}\partial_{t} y-\Delta y=f+\left.1\right|_{\omega} u & \text { in } \Omega \times(0, T), \\ y=0 & \text { on } \partial \Omega \times(0, T), \\ y(\cdot, 0)=y^{0} & \text { in } \Omega .\end{cases}
$$

For the moment, we choose $y^{0} \in L^{2}(\Omega)$ and $f \in L^{2}(\Omega \times(0, T))$.

Let $\left\{T_{k}\right\}_{k \geq 0}$ be the sequence of real positive numbers given by:

$$
T_{k}=T-\frac{T}{a^{k}},
$$

where $a>1$. Put $f_{k}=\left.1\right|_{\left(T_{k}, T_{k+1}\right)} f$. We start to describe the algorithm to construct the control: we initiate with $z_{0}=y^{0}$ and $w_{-1}=0$. Define the sequences $\left\{z_{k}\right\}_{k \geq 0},\left\{u_{k}\right\}_{k \geq 0}$, $\left\{v_{k}\right\}_{k \geq 0},\left\{w_{k}\right\}_{k \geq 0}$ as follows. Let $v_{k}$ be the solution of

$$
\begin{cases}\partial_{t} v_{k}-\Delta v_{k}=f_{k} & \text { in } \Omega \times\left(T_{k}, T_{k+1}\right), \\ v_{k}=0 & \text { on } \partial \times\left(T_{k}, T_{k+1}\right), \\ v_{k}\left(\cdot, T_{k}\right)=w_{k-1}\left(\cdot, T_{k}\right) & \text { in } \Omega .\end{cases}
$$

Introduce

$$
z_{k+1}=v_{k}\left(\cdot, T_{k+1}\right) .
$$

Let

$$
u_{k}=-C e^{\frac{C}{T_{k+1}-T_{k}}} \varphi_{k}
$$


where

$$
\begin{cases}\partial_{t} \varphi_{k}+\Delta \varphi_{k}=0 & \text { in } \Omega \times\left(T_{k}, T_{k+1}\right) \\ \varphi_{k}=0 & \text { on } \partial \Omega \times\left(T_{k}, T_{k+1}\right) \\ \varphi_{k}\left(\cdot, T_{k+1}\right)=\varphi_{k}^{T_{k+1}} & \text { in } \Omega\end{cases}
$$

Here $\varphi_{k}^{T_{k+1}}$ is the unique minimizer (see proof of Theorem 1.1, page 1399, [5]) of the following functional depending on $\epsilon_{k}>0: J_{\epsilon_{k}}: L^{2}(\Omega) \rightarrow \mathbb{R}$ given by

$$
J_{\epsilon_{k}}\left(\phi_{k}^{T_{k+1}}\right)=\frac{C e^{\frac{C}{T_{k+1}-T_{k}}}}{2} \int_{T_{k}}^{T_{k+1}} \int_{\omega}\left|\phi_{k}\right|^{2} d x d t+\frac{\epsilon_{k}}{2} \int_{\Omega}\left|\phi_{k}^{T_{k+1}}\right|^{2} d x+\int_{\Omega} \phi_{k}\left(\cdot, T_{k}\right) z_{k} d x,
$$

where $C$ is the constant in Theorem 2 and

$$
\begin{cases}\partial_{t} \phi_{k}+\Delta \phi_{k}=0 & \text { in } \Omega \times\left(T_{k}, T_{k+1}\right), \\ \phi_{k}=0 & \text { on } \partial \Omega \times\left(T_{k}, T_{k+1}\right), \\ \phi_{k}\left(\cdot, T_{k+1}\right)=\phi_{k}^{T_{k+1}} \in L^{2}(\Omega) . & \end{cases}
$$

Let $w_{k}$ be the solution of

$$
\begin{cases}\partial_{t} w_{k}-\Delta w_{k}=\left.1\right|_{\omega} u_{k} & \text { in } \Omega \times\left(T_{k}, T_{k+1}\right) \\ w_{k}=0 & \text { on } \partial \Omega \times\left(T_{k}, T_{k+1}\right) \\ w_{k}\left(\cdot, T_{k}\right)=z_{k} & \text { in } \Omega\end{cases}
$$

Therefore (see e.g. [5])

$$
w_{k}\left(\cdot, T_{k+1}\right)=\epsilon_{k} \varphi_{k}^{T_{k+1}} \quad \text { in } \quad \Omega
$$

and

$$
\frac{1}{C e^{\frac{C}{T_{k+1}-T_{k}}}} \int_{T_{k}}^{T_{k+1}} \int_{\omega}\left|u_{k}\right|^{2} d x d t+\frac{1}{\epsilon_{k}} \int_{\Omega}\left|w_{k}\left(\cdot, T_{k+1}\right)\right|^{2} d x \leq\left\|z_{k}\right\|_{L^{2}(\Omega)}^{2} .
$$

Finally, put $y_{k}=v_{k}+w_{k}$, then it solves

$$
\begin{cases}\partial_{t} y_{0}-\Delta y_{0}=f_{0}+\left.1\right|_{\omega} u_{0} & \text { in } \Omega \times\left(T, T_{1}\right) \\ y_{0}=0 & \text { on } \partial \Omega \times\left(T, T_{1}\right) \\ y_{0}(\cdot, 0)=y^{0} & \text { in } \Omega\end{cases}
$$

and

$$
\begin{cases}\partial_{t} y_{k+1}-\Delta y_{k+1}=f_{k+1}+\left.1\right|_{\omega} u_{k+1} & \text { in } \Omega \times\left(T_{k+1}, T_{k+2}\right), \\ y_{k+1}=0 & \text { on } \partial \Omega \times\left(T_{k+1}, T_{k+2}\right), \\ y_{k+1}\left(\cdot, T_{k+1}\right)=w_{k}\left(\cdot, T_{k+1}\right)+z_{k+1} & \text { in } \Omega .\end{cases}
$$

Notice that $y_{k}\left(\cdot, T_{k+1}\right)=y_{k+1}\left(\cdot, T_{k+1}\right)$, therefore the functions $y=\left.\sum_{k \geq 0} 1\right|_{\left[T_{k}, T_{k+1}\right]} y_{k}$ and $u=\left.\sum_{k \geq 0} 1\right|_{\left[T_{k}, T_{k+1}\right]} u_{k}$ satisfy $(3)$.

Now we are able to state our result: (recall that $a$ and $\epsilon_{k}$ are needed in (4) and (9) respectively). 
Theorem 4 Let $C$ be the constant in Theorem 2. There are $\lambda>0, a>1$ and $a$ sequence $\left\{\epsilon_{k}\right\}_{k \geq 0}$ of real positive numbers such that for any $y^{0} \in H_{0}^{1}(\Omega)$ and any $f \in$ $L^{2}(\Omega \times(0, T))$ such that $f e^{\frac{3 \lambda C}{T-t}} \in L^{2}(\Omega \times(0, T))$, the above constructed control function

$$
u=\left.\sum_{k \geq 0} 1\right|_{\left[T_{k}, T_{k+1}\right]} u_{k}
$$

is in $L^{2}(\omega \times(0, T))$ and drives the solution of (3) to $y(\cdot, T)=0$. Furthermore, there exists a positive constant $K$ such that the following estimate holds:

$$
\left\|\nabla y e^{\frac{\lambda C}{T-t}}\right\|_{C\left([0, T] ; L^{2}(\Omega)\right)} \leq K(1+\sqrt{T}) e^{\frac{3 \lambda C}{T}}\left\|\nabla y^{0}\right\|_{L^{2}(\Omega)}+K(1+T)\left\|f e^{\frac{3 \lambda C}{T-t}}\right\|_{L^{2}(\Omega \times(0, T))}
$$

Now, we come to the proof of Theorem 4.

\subsection{Proof of Theorem 4}

Our strategy to prove Theorem 4 is as follows: we want to get $\left\|y e^{\frac{M}{T-t}}\right\|_{C\left([0, T] ; L^{2}(\Omega)\right)}<+\infty$ for some suitable constant $M>0$ in order to deduce that $y(\cdot, T)=0$. To do so, since $y=\left.\sum_{k \geq 0} 1\right|_{\left[T_{k}, T_{k+1}\right]} y_{k}$ and $y_{k}=v_{k}+w_{k}$ is given by (5)-(8), we start to estimate $\left\|v_{k}\right\|_{C\left(\left[T_{k}, T_{k+1}\right] ; L^{2}(\Omega)\right)}$ and $\left\|w_{k}\right\|_{C\left(\left[T_{k}, T_{k+1}\right] ; L^{2}(\Omega)\right)}$. In same time, we also derive an inequality for $\left\|u e^{\frac{B}{T-t}}\right\|_{L^{2}(\omega \times(0, T))}$ for some suitable constant $B>0$ in order to get $u \in L^{2}(\omega \times(0, T))$. Finally, we will focus on estimating $\left\|\nabla y e^{\frac{D}{T-t}}\right\|_{C\left([0, T] ; L^{2}(\Omega)\right)}$ for some suitable constant $D>0$.

By the classical energy estimate for the heat equation with outside force, one has from (5)-(8)

$$
\begin{gathered}
\left\|v_{0}\right\|_{C\left(\left[T_{0}, T_{1}\right] ; L^{2}(\Omega)\right)} \leq \sqrt{T}\left\|f_{0}\right\|_{L^{2}\left(\Omega \times\left(T_{0}, T_{1}\right)\right)} \\
\left\|v_{k+1}\right\|_{C\left(\left[T_{k+1}, T_{k+2}\right] ; L^{2}(\Omega)\right)} \leq \sqrt{T}\left\|f_{k+1}\right\|_{L^{2}\left(\Omega \times\left(T_{k+1}, T_{k+2}\right)\right)}+\left\|w_{k}\left(\cdot, T_{k+1}\right)\right\|_{L^{2}(\Omega)}
\end{gathered}
$$

and

$$
\left\|w_{k}\right\|_{C\left(\left[T_{k}, T_{k+1}\right] ; L^{2}(\Omega)\right)} \leq \sqrt{T}\left\|u_{k}\right\|_{L^{2}\left(\omega \times\left(T_{k}, T_{k+1}\right)\right)}+\left\|z_{k}\right\|_{L^{2}(\Omega)} .
$$

By using the following estimates, which implied by (10):

$$
\left\|u_{k}\right\|_{L^{2}\left(\omega \times\left(T_{k}, T_{k+1}\right)\right)} \leq \sqrt{C} e^{\frac{C}{2} \frac{1}{T_{k+1}-T_{k}}}\left\|z_{k}\right\|_{L^{2}(\Omega)} \text { and }\left\|w_{k}\left(\cdot, T_{k+1}\right)\right\|_{L^{2}(\Omega)} \leq \sqrt{\epsilon_{k}}\left\|z_{k}\right\|_{L^{2}(\Omega)},
$$

we get

$$
\left\|v_{k+1}\right\|_{C\left(\left[T_{k+1}, T_{k+2}\right] ; L^{2}(\Omega)\right)} \leq \sqrt{T}\left\|f_{k+1}\right\|_{L^{2}\left(\Omega \times\left(T_{k+1}, T_{k+2}\right)\right)}+\sqrt{\epsilon_{k}}\left\|z_{k}\right\|_{L^{2}(\Omega)}
$$

and

$$
\left\|w_{k}\right\|_{C\left(\left[T_{k}, T_{k+1}\right] ; L^{2}(\Omega)\right)} \leq \sqrt{C T} e^{\frac{C}{2} \frac{1}{T_{k+1}-T_{k}}}\left\|z_{k}\right\|_{L^{2}(\Omega)}+\left\|z_{k}\right\|_{L^{2}(\Omega)} .
$$


Since by $(6) v_{k+1}\left(\cdot, T_{k+2}\right)=z_{k+2}$, it implies using (12) that

$$
\left\|z_{k+2}\right\|_{L^{2}(\Omega)} \leq \sqrt{T}\left\|f_{k+1}\right\|_{L^{2}\left(\Omega \times\left(T_{k+1}, T_{k+2}\right)\right)}+\sqrt{\epsilon_{k}}\left\|z_{k}\right\|_{L^{2}(\Omega)} .
$$

As a result, for any constant $A>0$, we get

$$
\begin{aligned}
& \sum_{k \geq 0} e^{\frac{A}{T-T_{k+1}}}\left\|z_{k}\right\|_{L^{2}(\Omega)} \\
& =e^{\frac{A}{T-T_{1}}}\left\|z_{0}\right\|_{L^{2}(\Omega)}+e^{\frac{A}{T-T_{2}}}\left\|z_{1}\right\|_{L^{2}(\Omega)}+\sum_{k \geq 0} e^{\frac{A}{T-T_{k+3}}}\left\|z_{k+2}\right\|_{L^{2}(\Omega)} \\
& \leq e^{\frac{A}{T-T_{1}}}\left\|y^{0}\right\|_{L^{2}(\Omega)}+e^{\frac{A}{T-T_{2}}} \sqrt{T}\left\|f_{0}\right\|_{L^{2}\left(\Omega \times\left(0, T_{1}\right)\right)} \\
& +\sqrt{T} \sum_{k \geq 0} e^{\frac{A}{T-T_{k+3}}}\left\|f_{k+1}\right\|_{L^{2}\left(\Omega \times\left(T_{k+1}, T_{k+2}\right)\right)}+\sum_{k \geq 0} e^{\frac{A}{T-T_{k+3}}} \sqrt{\epsilon_{k}}\left\|z_{k}\right\|_{L^{2}(\Omega)} \\
& \leq e^{\frac{A}{T-T_{1}}}\left\|y^{0}\right\|_{L^{2}(\Omega)}+\sqrt{T} \sum_{k \geq 0} e^{\frac{A}{T-T_{k+2}}}\left\|f_{k}\right\|_{L^{2}\left(\Omega \times\left(T_{k}, T_{k+1}\right)\right)}+\sum_{k \geq 0} e^{\frac{A}{T-T_{k+3}}} \sqrt{\epsilon_{k}}\left\|z_{k}\right\|_{L^{2}(\Omega)} \\
& \leq e^{\frac{A}{T-T_{1}}}\left\|y^{0}\right\|_{L^{2}(\Omega)}+\sqrt{T} \sum_{k \geq 0} e^{\frac{a A}{T-T_{k+1}}}\left\|f_{k}\right\|_{L^{2}\left(\Omega \times\left(T_{k}, T_{k+1}\right)\right)}+\sum_{k \geq 0} e^{\frac{a^{2} A}{T-T_{k+1}}} \sqrt{\epsilon_{k}}\left\|z_{k}\right\|_{L^{2}(\Omega)} .
\end{aligned}
$$

Choose

$$
\epsilon_{k}=\frac{1}{4} e^{-\frac{2 A\left(a^{2}-1\right)}{T-T_{k+1}}}
$$

in order that $e^{\frac{a^{2} A}{T-T_{k+1}}} \sqrt{\epsilon_{k}} \leq \frac{1}{2} e^{\frac{A}{T-T_{k+1}}}$, then (14) becomes

$$
\sum_{k \geq 0} e^{\frac{A}{T-T_{k+1}}}\left\|z_{k}\right\|_{L^{2}(\Omega)} \leq 2 e^{\frac{A}{T-T_{1}}}\left\|y^{0}\right\|_{L^{2}(\Omega)}+2 \sqrt{T} \sum_{k \geq 0} e^{\frac{a A}{T-T_{k+1}}}\left\|f_{k}\right\|_{L^{2}\left(\Omega \times\left(T_{k}, T_{k+1}\right)\right)} .
$$

On one hand, for any constant $M>0$, we obtain by (12), (13) and (15)

$$
\begin{aligned}
& \sum_{k \geq 0} e^{\frac{M}{T-T_{k+1}}}\left\|y_{k}\right\|_{C\left(\left[T_{k}, T_{k+1}\right] ; L^{2}(\Omega)\right)} \\
& \leq \sum_{k \geq 0} e^{\frac{M}{T-T_{k+1}}}\left\|v_{k}\right\|_{C\left(\left[T_{k}, T_{k+1}\right] ; L^{2}(\Omega)\right)}+\sum_{k \geq 0} e^{\frac{M}{T-T_{k+1}}}\left\|w_{k}\right\|_{C\left(\left[T_{k}, T_{k+1}\right] ; L^{2}(\Omega)\right)} \\
& \leq e^{\frac{M}{T-T_{1}}} \sqrt{T}\left\|f_{0}\right\|_{L^{2}\left(\Omega \times\left(T_{0}, T_{1}\right)\right)}+\sum_{k \geq 1} e^{\frac{M}{T-T_{k+1}}} \sqrt{T}\left\|f_{k}\right\|_{L^{2}\left(\Omega \times\left(T_{k}, T_{k+1}\right)\right)} \\
& +\sum_{k \geq 0} e^{\frac{M}{T-T_{k+2}}} \sqrt{\epsilon_{k}}\left\|z_{k}\right\|_{L^{2}(\Omega)}+\sum_{k \geq 0} e^{\frac{M}{T-T_{k+1}}}\left(1+\sqrt{C T} e^{\frac{C}{2} \frac{1}{T_{k+1}-T_{k}}}\right)\left\|z_{k}\right\|_{L^{2}(\Omega)} \\
& \leq \sum_{k \geq 0} e^{\frac{M}{T-T_{k+1}}} \sqrt{T}\left\|f_{k}\right\|_{L^{2}\left(\Omega \times\left(T_{k}, T_{k+1}\right)\right)} \\
& +\frac{1}{2} \sum_{k \geq 0} e^{\frac{a M-A\left(a^{2}-1\right)}{T-T_{k+1}}}\left\|z_{k}\right\|_{L^{2}(\Omega)}+\sum_{k \geq 0} e^{\frac{M}{T-T_{k+1}}}\left\|z_{k}\right\|_{L^{2}(\Omega)} \\
& +\sqrt{C T} \sum_{k \geq 0} e^{\left(M+\frac{C}{2(a-1)}\right) \frac{1}{T-T_{k+1}}}\left\|z_{k}\right\|_{L^{2}(\Omega)} \\
& \leq \sum_{k \geq 0} e^{\frac{M}{T-T_{k+1}}} \sqrt{T}\left\|f_{k}\right\|_{L^{2}\left(\Omega \times\left(T_{k}, T_{k+1}\right)\right)}+\frac{3}{2}(1+\sqrt{C T}) \sum_{k \geq 0} e^{\frac{N}{T-T_{k+1}}}\left\|z_{k}\right\|_{L^{2}(\Omega)},
\end{aligned}
$$


where $N=\max \left\{a M-A\left(a^{2}-1\right), M, M+\frac{C}{2(a-1)}\right\}$, which implies under the condition $N \leq A$ with (16) that

$$
\begin{aligned}
\sum_{k \geq 0} e^{\frac{M}{T-T_{k+1}}}\left\|y_{k}\right\|_{C\left(\left[T_{k}, T_{k+1}\right] ; L^{2}(\Omega)\right)} \leq & 3(1+\sqrt{C T}) e^{\frac{A}{T-T_{1}}}\left\|y^{0}\right\|_{L^{2}(\Omega)} \\
& +3 \sqrt{T}(1+\sqrt{C T}) \sum_{k \geq 0} e^{\frac{a A}{T-T_{k+1}}}\left\|f_{k}\right\|_{L^{2}\left(\Omega \times\left(T_{k}, T_{k+1}\right)\right)} .
\end{aligned}
$$

Therefore

$$
\begin{aligned}
& \left\|y e^{\frac{M}{T-t}}\right\|_{C\left([0, T] ; L^{2}(\Omega)\right)} \\
\leq & \sum_{k \geq 0} e^{\frac{M}{T-T_{k+1}}}\left\|y_{k}\right\|_{C\left(\left[T_{k}, T_{k+1}\right] ; L^{2}(\Omega)\right)} \\
\leq & 3(1+\sqrt{C T})\left(e^{\frac{A}{T-T_{1}}}\left\|y^{0}\right\|_{L^{2}(\Omega)}\right)+3 \sqrt{T}(1+\sqrt{C T})\left\|f e^{\frac{a^{2} A}{T-t}}\right\|_{L^{2}(\Omega \times(0, T))} .
\end{aligned}
$$

On the other hand, by the first inequality in (11), one has for any constant $B>0$

$$
\begin{aligned}
\sum_{k \geq 0} e^{\frac{B}{T-T_{k+1}}}\left\|u_{k}\right\|_{L^{2}\left(\omega \times\left(T_{k}, T_{k+1}\right)\right)} & \leq \sum_{k \geq 0} e^{\frac{B}{T-T_{k+1}}} \sqrt{C} e^{\frac{C}{2} \frac{1}{T_{k+1}-T_{k}}}\left\|z_{k}\right\|_{L^{2}(\Omega)} \\
& \leq \sqrt{C} \sum_{k \geq 0} e^{\left(B+\frac{C}{2(a-1)}\right) \frac{1}{T-T_{k+1}}}\left\|z_{k}\right\|_{L^{2}(\Omega)},
\end{aligned}
$$

which implies under the condition $B+\frac{C}{2(a-1)} \leq A$ with (16), that

$$
\begin{aligned}
\sum_{k \geq 0} e^{\frac{B}{T-T_{k+1}}}\left\|u_{k}\right\|_{L^{2}\left(\omega \times\left(T_{k}, T_{k+1}\right)\right) \leq} & 2 \sqrt{C} e^{\frac{A}{T-T_{1}}}\left\|y^{0}\right\|_{L^{2}(\Omega)} \\
& +2 \sqrt{C T} \sum_{k \geq 0} e^{\frac{a A}{T-T_{k+1}}}\left\|f_{k}\right\|_{L^{2}\left(\Omega \times\left(T_{k}, T_{k+1}\right)\right)} .
\end{aligned}
$$

Therefore

$$
\begin{aligned}
\left\|u e^{\frac{B}{T-t}}\right\|_{L^{2}(\omega \times(0, T))} & \leq \sum_{k \geq 0} e^{\frac{B}{T-T_{k+1}}}\left\|u_{k}\right\|_{L^{2}\left(\omega \times\left(T_{k}, T_{k+1}\right)\right)} \\
& \leq 2 \sqrt{C} e^{\frac{A}{T-T_{1}}}\left\|y^{0}\right\|_{L^{2}(\Omega)}+2 \sqrt{C T}\left\|f e^{\frac{a^{2} A}{T-t}}\right\|_{L^{2}(\Omega \times(0, T))} .
\end{aligned}
$$

By taking $B=M=\frac{C}{2(a-1)}$ and $A=\frac{C}{a-1}$, we conclude from (17) and (18) that

$$
\begin{aligned}
& \left\|y e^{\frac{C}{2(a-1)} \frac{1}{T-t}}\right\|_{C\left([0, T] ; L^{2}(\Omega)\right)}+\left\|u e^{\frac{C}{2(a-1)} \frac{1}{T-t}}\right\|_{L^{2}(\omega \times(0, T))} \\
\leq & c(1+\sqrt{T}) e^{\frac{a C}{a-1} \frac{1}{T}}\left\|y^{0}\right\|_{L^{2}(\Omega)}+c \sqrt{T}(1+\sqrt{T})\left\|f e^{\frac{a^{2} C}{a-1} \frac{1}{T-t}}\right\|_{L^{2}(\Omega \times(0, T))},
\end{aligned}
$$

for some constant $c$. We turn now to the case $y^{0} \in H_{0}^{1}(\Omega)$. For any constant $D>0$, put $p=p(t)=e^{\frac{D}{T-t}}$ and $g=p y$ then $g$ satisfies the following system

$$
\begin{cases}\partial_{t} g-\Delta g=p^{\prime} y+p\left(\left.1\right|_{\omega} u-f\right) & \text { in } \Omega \times(0, T), \\ g=0 & \text { on } \partial \Omega \times(0, T), \\ g(\cdot, 0)=e^{\frac{D}{T}} y^{0} & \text { in } \Omega .\end{cases}
$$


Applying classical energy estimate, one has

$\|\nabla g\|_{C\left([0, T] ; L^{2}(\Omega)\right)} \leq e^{\frac{D}{T}}\left\|\nabla y^{0}\right\|_{L^{2}(\Omega)}+\left\|p^{\prime} y\right\|_{L^{2}(\Omega \times(0, T))}+\|p u\|_{L^{2}(\Omega \times(0, T))}+\|p f\|_{L^{2}(\Omega \times(0, T))}$, which implies, for any $\rho \in(1,3 / 2)$ the existence of $K_{\rho}>0$ such that

$$
\begin{aligned}
\left\|\nabla y e^{\frac{D}{T-t}}\right\|_{C\left([0, T] ; L^{2}(\Omega)\right) \leq} \leq & e^{\frac{D}{T}}\left\|\nabla y^{0}\right\|_{L^{2}(\Omega)}+K_{\rho}\left\|y e^{\frac{\rho D}{T-t}}\right\|_{L^{2}(\Omega \times(0, T))} \\
& +\left\|u e^{\frac{\rho D}{T-t}}\right\|_{L^{2}(\Omega \times(0, T))}+\left\|f e^{\frac{3 D}{T-t}}\right\|_{L^{2}(\Omega \times(0, T))} .
\end{aligned}
$$

Take

$$
a=\sqrt{\frac{3}{2 \rho}} \text { and } D=\frac{C}{2 \rho\left(\sqrt{\frac{3}{2 \rho}}-1\right)},
$$

in order that $a>1, \rho D=\frac{C}{2(a-1)}$ and $\frac{a^{2} C}{a-1}=3 D$. Then, it implies by combining (19) and (20) that

$$
\left\|\nabla y e^{\frac{D}{T-t}}\right\|_{C\left([0, T] ; L^{2}(\Omega)\right)} \leq K(1+\sqrt{T}) e^{\frac{3 D}{T}}\left\|\nabla y^{0}\right\|_{L^{2}(\Omega)}+K(1+T)\left\|f e^{\frac{3 D}{T-t}}\right\|_{L^{2}(\Omega \times(0, T))},
$$

for some constant $K$. With $\lambda=\frac{1}{2 \rho\left(\sqrt{\frac{3}{2 \rho}}-1\right)}$ in order that $D=\lambda C$, we have completed the proof of Theorem 4 .

\section{Proof of main results}

This section focuses on the proof of the main results, Theorem 1 and Corollary 1, which ensures that system (1) is null controllable with the different conditions of the initial data. First, we start with the proof of Theorem 1.

\subsection{Proof of Theorem 1}

The idea of the proof of Theorem 1 is as follows: first, by applying the result in Theorem 4 , we construct a control sequence $u_{m} \in L^{2}(\Omega \times(0, T))$ such that the solution of

$$
\begin{cases}\partial_{t} y_{m}-\Delta y_{m}+\gamma y_{m-1}^{3}=\left.1\right|_{\omega} u_{m} & \text { in } \Omega \times(0, T) \\ y_{m}=0 & \text { on } \partial \Omega \times(0, T) \\ y_{m}(\cdot, 0)=y^{0} & \text { in } \Omega\end{cases}
$$

satisfies $y_{m}(\cdot, T)=0$ in $H_{0}^{1}(\Omega)$; secondly, by proving $y_{m}$ converges to $y$ and $u_{m}$ converges to $u$, we will get the desired result. Now, we start the first step by checking that the function $f=-\gamma y_{m-1}^{3}$ satisfies the condition of Theorem 4. Denote $D=\lambda C$. First take $y_{0}$ such that $y_{0}(\cdot, 0)=y^{0}$ and $\gamma y_{0}^{3} e^{\frac{3 D}{T-t}} \in L^{2}(\Omega \times(0, T))$, for example $y_{0}=e^{-\frac{D}{T-t}} e^{\frac{D}{T}} y^{0}$. 
Now by induction, we will prove that $\gamma y_{m}^{3} e^{\frac{3 D}{T-t}} \in L^{2}(\Omega \times(0, T))$ for any $m \geq 1$. Indeed, suppose $y_{m-1}^{3} e^{\frac{3 D}{T-t}} \in L^{2}(\Omega \times(0, T))$, by Theorem $4, y_{m}$ verifies

$\left\|\nabla y_{m}(\cdot, t) e^{\frac{D}{T-t}}\right\|_{L^{2}(\Omega)} \leq K(1+\sqrt{T}) e^{\frac{3 D}{T}}\left\|\nabla y^{0}\right\|_{L^{2}(\Omega)}+K(1+T)\left\|y_{m-1}^{3} e^{\frac{3 D}{T-t}}\right\|_{L^{2}(\Omega \times(0, T))}$.

Using Sobolev embedding, we obtain

$$
\begin{aligned}
& \left\|y_{m}^{3} e^{\frac{3 D}{T-t}}\right\|_{L^{2}(\Omega \times(0, T))}^{2} \\
& \leq c \int_{0}^{T}\left\|\nabla y_{m}(\cdot, t) e^{\frac{D}{T-t}}\right\|_{L^{2}(\Omega)}^{6} d t \\
& \leq c K T\left((1+\sqrt{T}) e^{\frac{K}{T}}\left\|\nabla y^{0}\right\|_{L^{2}(\Omega)}+(1+T)\left\|y_{m-1}^{3} e^{\frac{3 D}{T-t}}\right\|_{L^{2}(\Omega \times(0, T))}\right)^{6} \\
& <\infty
\end{aligned}
$$

from the induction assumption. Thus, the control $u_{m}$ constructed in Theorem 4 leads to $y_{m}(\cdot, T)=0$, which completes the first step. Now, we pass to the second step by proving that $\left\{y_{m}(\cdot, t) e^{\frac{D}{T-t}}\right\}$ is bounded in $C\left([0, T], H_{0}^{1}(\Omega)\right)$ for any $m \geq 1$. From the inequality in Theorem 4 with $D=\lambda C$ (or simply (22)) and Sobolev embedding, we get

$$
\begin{aligned}
& \left\|\nabla y_{m}(\cdot, t) e^{\frac{D}{T-t}}\right\|_{L^{2}(\Omega)} \\
\leq & K(1+\sqrt{T}) e^{\frac{3 D}{T}}\left\|\nabla y^{0}\right\|_{L^{2}(\Omega)}+c K(1+T)\left(\int_{0}^{T}\left\|\nabla y_{m-1}(\cdot, t) e^{\frac{D}{T-t}}\right\|_{L^{2}(\Omega)}^{6} d t\right)^{\frac{1}{2}} \\
\leq & K(1+\sqrt{T}) e^{\frac{3 D}{T}}\left\|\nabla y^{0}\right\|_{L^{2}(\Omega)}+c K \sqrt{T}(1+T)\left(\sup _{t \in[0, T]}\left\|\nabla y_{m-1}(\cdot, t) e^{\frac{D}{T-t}}\right\|_{L^{2}(\Omega)}\right)^{3},
\end{aligned}
$$

which implies

$$
\begin{aligned}
& \sup _{t \in[0, T]}\left\|\nabla y_{m} e^{\frac{D}{T-t}}\right\|_{L^{2}(\Omega)} \\
\leq & K(1+\sqrt{T}) e^{\frac{3 D}{T}}\left\|\nabla y^{0}\right\|_{L^{2}(\Omega)}+c K \sqrt{T}(1+T)\left(\sup _{t \in[0, T]}\left\|\nabla y_{m-1} e^{\frac{D}{T-t}}\right\|_{L^{2}(\Omega)}\right)^{3} .
\end{aligned}
$$

Therefore, if

$$
8\left(K(1+\sqrt{T}) e^{\frac{3 D}{T}}\left\|\nabla y^{0}\right\|_{L^{2}(\Omega)}\right)^{2} \leq \frac{1}{c K \sqrt{T}(1+T)}
$$

and

$$
e^{\frac{3 D}{T}}\left\|\nabla y^{0}\right\|_{L^{2}(\Omega)}=\sup _{t \in[0, T]}\left\|\nabla y_{0} e^{\frac{3 D}{T-t}}\right\|_{L^{2}(\Omega)} \leq 2 K(1+\sqrt{T}) e^{\frac{3 D}{T}}\left\|\nabla y^{0}\right\|_{L^{2}(\Omega)}
$$

then by induction, we have for any $m \geq 1$

$$
\sup _{t \in[0, T]}\left\|\nabla y_{m} e^{\frac{3 D}{T-t}}\right\|_{L^{2}(\Omega)} \leq 2 K(1+\sqrt{T}) e^{\frac{3 D}{T}}\left\|\nabla y^{0}\right\|_{L^{2}(\Omega)}
$$

Thus, $y_{m} e^{\frac{D}{T-t}}$ is bounded in $C\left([0, T] ; H_{0}^{1}(\Omega)\right)$ for any $m \geq 1$, whenever $\left\|y^{0}\right\|_{H_{1}^{0}(\Omega)}$ is sufficiently small. Now we prove that $\left\{y_{m} e^{\frac{D}{T-t}}\right\}$ and $\left\{u_{m} e^{\frac{D}{T-t}}\right\}$ are Cauchy sequences in 
$C\left([0, T], H_{0}^{1}(\Omega)\right)$ and $L^{2}(\omega \times(0, T))$, respectively, for any $m \geq 1$. Indeed, put $Y_{m+1}=$ $y_{m+1}-y_{m}$ and $U_{m+1}=u_{m+1}-u_{m}$ for any $m \geq 1$ then $Y_{m+1}$ is solution of

$$
\begin{cases}\partial_{t} Y_{m+1}-\Delta Y_{m+1}=-\gamma\left(y_{m}^{3}-y_{m-1}^{3}\right)+\left.1\right|_{\omega} U_{m+1} & \text { in } \Omega \times(0, T), \\ Y_{m+1}=0 & \text { on } \partial \Omega \times(0, T), \\ Y_{m+1}(\cdot, 0)=0 & \text { in } \Omega .\end{cases}
$$

Firstly, we will estimate $U_{m+1} e^{\frac{D}{T-t}}$. Recall that the control function $u_{m}$ is constructed by

$$
u_{m}=\sum_{k \geq 0} u_{m, k}=-\sum_{k \geq 0} C e^{\frac{C}{T_{k+1}-T_{k}}} \varphi_{m, k},
$$

where $\varphi_{m+1, k}-\varphi_{m, k}$ solves

$$
\begin{cases}\partial_{t}\left(\varphi_{m+1, k}-\varphi_{m, k}\right)+\Delta\left(\varphi_{m+1, k}-\varphi_{m, k}\right)=0 & \text { in } \quad \Omega \times\left(T_{k}, T_{k+1}\right), \\ \left(\varphi_{m+1, k}-\varphi_{m, k}\right)=0 & \text { on } \quad \partial \Omega \times\left(T_{k}, T_{k+1}\right), \\ \left(\varphi_{m+1, k}-\varphi_{m, k}\right)\left(\cdot, T_{k+1}\right) \in L^{2}(\Omega) . & \end{cases}
$$

Furthermore, we also have constructed the functions $w_{m+1, k}$ and $w_{m, k}$ by applying Theorem 4 with $f=-\gamma y_{m}^{3}$ and $f=-\gamma y_{m-1}^{3}$ respectively:

$$
\begin{cases}\partial_{t}\left(w_{m+1, k}-w_{m, k}\right)-\Delta\left(w_{m+1, k}-w_{m, k}\right) & \text { in } \Omega \times\left(T_{k}, T_{k+1}\right), \\ =-C e^{\frac{C}{T_{k+1} T_{k}}}\left(\varphi_{m+1, k}-\varphi_{m, k}\right) & \text { on } \partial \Omega \times\left(T_{k}, T_{k+1}\right), \\ \left(w_{m+1, k}-w_{m, k}\right)=0 & \text { in } \Omega, \\ \left(w_{m+1, k}-w_{m, k}\right)\left(\cdot, T_{k}\right)=z_{m+1, k}-z_{m, k} & \text { in } \Omega .\end{cases}
$$

Multiplying the two sides of the first equation in (24) by $\left(\varphi_{m+1, k}-\varphi_{m, k}\right)$ and integrating over $\Omega \times\left(T_{k}, T_{k+1}\right)$, we get

$$
\begin{aligned}
& -C e^{\frac{C}{T_{k+1}-T_{k}}} \int_{T_{k}}^{T_{k+1}} \int_{\omega}\left|\varphi_{m+1, k}-\varphi_{m, k}\right|^{2} d x d t \\
= & \int_{\Omega}\left(w_{m+1, k}-w_{m, k}\right)\left(\cdot, T_{k+1}\right)\left(\varphi_{m+1, k}-\varphi_{m, k}\right)\left(\cdot, T_{k+1}\right) d x \\
& -\int_{\Omega}\left(w_{m+1, k}-w_{m, k}\right)\left(\cdot, T_{k}\right)\left(\varphi_{m+1, k}-\varphi_{m, k}\right)\left(\cdot, T_{k}\right) d x \\
= & \epsilon_{k} \int_{\Omega}\left|\left(\varphi_{m+1, k}-\varphi_{m, k}\right)\left(\cdot, T_{k+1}\right)\right|^{2} d x-\int_{\Omega}\left(z_{m+1, k}-z_{m, k}\right)\left(\varphi_{m+1, k}-\varphi_{m, k}\right)\left(\cdot, T_{k}\right) d x .
\end{aligned}
$$

Therefore, we can write

$$
\begin{aligned}
& C e^{\frac{C}{T_{k+1}-T_{k}}} \int_{T_{k}}^{T_{k+1}} \int_{\omega}\left|\varphi_{m+1, k}-\varphi_{m, k}\right|^{2} d x d t+\epsilon_{k} \int_{\Omega}\left|\left(\varphi_{m+1, k}-\varphi_{m, k}\right)\left(\cdot, T_{k+1}\right)\right|^{2} d x \\
= & \int_{\Omega}\left(z_{m+1, k}-z_{m, k}\right)\left(\varphi_{m+1, k}-\varphi_{m, k}\right)\left(\cdot, T_{k}\right) d x \\
\leq & \left\|z_{m+1, k}-z_{m, k}\right\|_{L^{2}(\Omega)}\left\|\left(\varphi_{m+1, k}-\varphi_{m, k}\right)\left(\cdot, T_{k}\right)\right\|_{L^{2}(\Omega)} .
\end{aligned}
$$


By the classical observability estimate (see Theorem 3), we deduce that

$$
\begin{aligned}
& C e^{\frac{C}{T_{k+1}-T_{k}}} \int_{T_{k}}^{T_{k+1}} \int_{\omega}\left|\varphi_{m+1, k}-\varphi_{m, k}\right|^{2} d x d t \\
\leq & \left\|z_{m+1, k}-z_{m, k}\right\|_{L^{2}(\Omega)}\left(C e^{\frac{C}{T_{k+1}-T_{k}}} \int_{T_{k}}^{T_{k+1}} \int_{\omega}\left|\varphi_{m+1, k}-\varphi_{m, k}\right|^{2} d x d t\right)^{\frac{1}{2}} .
\end{aligned}
$$

Therefore, we get

$$
\sqrt{C} e^{\frac{C}{2} \frac{1}{T_{k+1}-T_{k}}}\left\|\varphi_{m+1, k}-\varphi_{m, k}\right\|_{L^{2}\left(\omega \times\left(T_{k}, T_{k+1}\right)\right)} \leq\left\|z_{m+1, k}-z_{m, k}\right\|_{L^{2}(\Omega)} .
$$

As a result

$$
\begin{aligned}
\left\|U_{m+1} e^{\frac{D}{T-t}}\right\|_{L^{2}(\Omega \times(0, T))} & =\left\|\sum_{k \geq 0}\left(u_{m+1, k}-u_{m, k}\right) e^{\frac{D}{T-t}}\right\|_{L^{2}\left(\Omega \times\left(T_{k}, T_{k+1}\right)\right)} \\
& =\left\|\sum_{k \geq 0} C e^{\frac{C}{T_{k+1}-T_{k}}} e^{\frac{D}{T-t}}\left(\varphi_{m+1, k}-\varphi_{m, k}\right)\right\|_{L^{2}\left(\Omega \times\left(T_{k}, T_{k+1}\right)\right)} \\
& \leq \sum_{k \geq 0} C e^{\frac{C}{T_{k+1}-T_{k}}} e^{\frac{D}{T-T_{k+1}}}\left\|\varphi_{m+1, k}-\varphi_{m, k}\right\|_{L^{2}\left(\Omega \times\left(T_{k}, T_{k+1}\right)\right)} \\
& \leq \sqrt{C} \sum_{k \geq 0} e^{\left(D+\frac{C}{2(a-1)}\right) \frac{1}{T-T_{k+1}}}\left\|z_{m+1, k}-z_{m, k}\right\|_{L^{2}\left(\Omega \times\left(T_{k}, T_{k+1}\right)\right)} .
\end{aligned}
$$

Recall that the constants $a$ and $D$ were given in the proof of Theorem 4 (see (21)) and satisfy $D+\frac{C}{2(a-1)}=\frac{C}{2 \rho(a-1)}+\frac{C}{2(a-1)} \leq \frac{C}{(a-1)}$. Following the same computations as in the proof of Theorem 4 (see (16)), we get

$$
\begin{aligned}
\sum_{k \geq 0} e^{\frac{C}{a-1} \frac{1}{T-T_{k+1}}}\left\|z_{m+1, k}-z_{m, k}\right\|_{L^{2}(\Omega)} & \leq 2 \sqrt{T} \sum_{k \geq 0} e^{\frac{a C}{a-1} \frac{1}{T-T_{k+1}}}\left\|y_{m, k}^{3}-y_{m-1, k}^{3}\right\|_{L^{2}\left(\Omega \times\left(T_{k}, T_{k+1}\right)\right)} \\
& \leq 2 \sqrt{T}\left\|\left(y_{m}^{3}-y_{m-1}^{3}\right) e^{\frac{3 D}{T-t}}\right\|_{L^{2}(\Omega \times(0, T)} .
\end{aligned}
$$

Therefore, combining (25) and (26), it holds:

$$
\left\|U_{m+1} e^{\frac{D}{T-t}}\right\|_{L^{2}(\omega \times(0, T))} \leq 2 \sqrt{C T}\left\|\left(y_{m}^{3}-y_{m-1}^{3}\right) e^{\frac{3 D}{T-t}}\right\|_{L^{2}(\Omega \times(0, T)} .
$$

Secondly, following the same computations than in the proof of Theorem 4 (see (22)), we obtain

$$
\left\|\nabla Y_{m+1}(\cdot, t) e^{\frac{D}{T-t}}\right\|_{L^{2}(\Omega)} \leq K(1+T)\left\|\left(y_{m}^{3}-y_{m-1}^{3}\right) e^{\frac{3 D}{T-t}}\right\|_{L^{2}(\Omega \times(0, T))} \quad \forall t \in(0, T) .
$$

But, from $\left|a^{3}-b^{3}\right| \leq 2|a-b|(a+b)^{2}$ and Hölder inequality, we have:

$$
\begin{aligned}
\left\|y_{m}^{3}-y_{m-1}^{3}\right\|_{L^{2}(\Omega)}^{2} & \leq 4 \int_{\Omega}\left(y_{m}-y_{m-1}\right)^{2}\left(y_{m}+y_{m-1}\right)^{4} d x \\
& \leq 4\left(\int_{\Omega}\left(y_{m}-y_{m-1}\right)^{6}\right)^{\frac{1}{3}}\left(\int_{\Omega}\left(y_{m}+y_{m-1}\right)^{6}\right)^{\frac{2}{3}} \\
& \leq c\left\|\nabla y_{m}-\nabla y_{m-1}\right\|_{L^{2}(\Omega)}^{2}\left\|\nabla y_{m}+\nabla y_{m-1}\right\|_{L^{2}(\Omega)}^{4} \\
& \leq 4 c\left\|\nabla Y_{m}\right\|_{L^{2}(\Omega)}^{2}\left(\left\|\nabla y_{m}\right\|_{L^{2}(\Omega)}^{2}+\left\|\nabla y_{m-1}\right\|_{L^{2}(\Omega)}^{2}\right)^{2}
\end{aligned}
$$


As a result,

$$
\begin{aligned}
& \left\|\left(y_{m}^{3}-y_{m-1}^{3}\right) e^{\frac{3 D}{T-t}}\right\|_{L^{2}(\Omega \times(0, T))}=\left(\int_{0}^{T}\left\|\left(y_{m}^{3}-y_{m-1}^{3}\right)\right\|_{L^{2}(\Omega)}^{2} e^{\frac{6 D}{T-t}} d t\right)^{\frac{1}{2}} \\
\leq & \left(\int_{0}^{T} 4 c\left\|\nabla Y_{m}\right\|_{L^{2}(\Omega)}^{2}\left(\left\|\nabla y_{m}\right\|_{L^{2}(\Omega)}^{2}+\left\|\nabla y_{m-1}\right\|_{L^{2}(\Omega)}^{2}\right)^{2} e^{\frac{6 D}{T-t}} d t\right)^{\frac{1}{2}} \\
\leq & \left(\int_{0}^{T} 4 c\left\|\nabla Y_{m} e^{\frac{D}{T-t}}\right\|_{L^{2}(\Omega)}^{2}\left(\left\|\nabla y_{m} e^{\frac{D}{T-t}}\right\|_{L^{2}(\Omega)}^{2}+\left\|\nabla y_{m-1} e^{\frac{D}{T-t}}\right\|_{L^{2}(\Omega)}^{2}\right)^{2} d t\right)^{\frac{1}{2}} .
\end{aligned}
$$

So by (23), it implies:

$$
\begin{aligned}
& \left\|\left(y_{m}^{3}-y_{m-1}^{3}\right) e^{\frac{3 D}{T-t}}\right\|_{L^{2}(\Omega \times(0, T))} \\
& \leq 4 \sqrt{T} c \sup _{t \in[0, T]}\left\|\nabla Y_{m} e^{\frac{D}{T-t}}\right\|_{L^{2}(\Omega)}\left(\sup _{t \in[0, T]}\left\|\nabla y_{m} e^{\frac{D}{T-t}}\right\|_{L^{2}(\Omega)}\right)^{2} \\
& \leq 4 \sqrt{T} c \sup _{t \in[0, T]}\left\|\nabla Y_{m} e^{\frac{D}{T-t}}\right\|_{L^{2}(\Omega)}\left(2 K(1+\sqrt{T}) e^{\frac{3 D}{T}}\left\|\nabla y^{0}\right\|_{L^{2}(\Omega)}\right)^{2} .
\end{aligned}
$$

Gathering (28), (27) and (29), yields:

$$
\begin{aligned}
& \left\|\nabla Y_{m+1}(\cdot, t) e^{\frac{D}{T-t}}\right\|_{L^{2}(\Omega)} \\
\leq & K(1+T)\left\|\left(y_{m}^{3}-y_{m-1}^{3}\right) e^{\frac{3 D}{T-t}}\right\|_{L^{2}(\Omega \times(0, T))} \\
\leq & 4 K(1+T) \sqrt{T} c \sup _{t \in[0, T]}\left\|\nabla Y_{m} e^{\frac{D}{T-t}}\right\|_{L^{2}(\Omega)}\left(2 K(1+\sqrt{T}) e^{\frac{3 D}{T}}\left\|\nabla y^{0}\right\|_{L^{2}(\Omega)}\right)^{2}
\end{aligned}
$$

and

$$
\begin{aligned}
& \left\|U_{m+1} e^{\frac{D}{T-t}}\right\|_{L^{2}(\omega \times(0, T))} \\
& \leq 2 \sqrt{C T}\left\|\left(y_{m}^{3}-y_{m-1}^{3}\right) e^{\frac{3 D}{T-t}}\right\|_{L^{2}(\Omega \times(0, T)} \\
& \leq 8 \sqrt{C T} T \sup _{t \in[0, T]}\left\|\nabla Y_{m} e^{\frac{D}{T-t}}\right\|_{L^{2}(\Omega)}\left(2 K(1+\sqrt{T}) e^{\frac{3 D}{T}}\left\|\nabla y^{0}\right\|_{L^{2}(\Omega)}\right)^{2} .
\end{aligned}
$$

Therefore, whenever

$$
4 K(1+T) \sqrt{T} c\left(2 K(1+\sqrt{T}) e^{\frac{3 D}{T}}\left\|\nabla y^{0}\right\|_{L^{2}(\Omega)}\right)^{2}<1
$$

which can be written

$$
\left\|y^{0}\right\|_{H_{0}^{1}(\Omega)}^{2} \leq \max _{[0 ; T]} \frac{1}{G(1+t)^{2} \sqrt{t} e^{\frac{G}{t}}}
$$

for some constant $G>1$, then $y_{m}$ converges to $y$ in $C\left([0, T], H_{0}^{1}(\Omega)\right)$ and $u_{m}$ converges to $u$ in $L^{2}(\omega \times(0, T))$. This completes the proof. 


\subsection{Proof of Corollary 1}

Now, we prove Corollary 1. Consider the following system:

$$
\begin{cases}\partial_{t} \widehat{y}-\Delta \widehat{y}+\widehat{y}^{3}=0 & \text { in } \Omega \times(0, T / 2), \\ \widehat{y}=0 & \text { on } \partial \Omega \times(0, T / 2), \\ \widehat{y}(\cdot, 0)=y^{0} & \text { in } \Omega .\end{cases}
$$

Recall that no blow-up phenomena occurs. We can establish by classical energy estimate that $\widehat{y}(\cdot, T / 2) \in H_{0}^{1}(\Omega)$. Furthermore, one has

$$
\|\widehat{y}(\cdot, T / 2)\|_{H_{0}^{1}(\Omega)}^{2} \leq \frac{1}{T}\left\|y^{0}\right\|_{L^{2}(\Omega)}^{2} \leq \max _{[0 ; T]} \frac{1}{G(1+t)^{2} \sqrt{t} e^{\frac{G}{t}}} .
$$

Consequently, applying Theorem 1, we obtain the existence of $\widetilde{u} \in L^{2}(\Omega \times(T / 2, T))$ such that the solution of

$$
\begin{cases}\partial_{t} \widetilde{y}-\Delta \widetilde{y}+\widetilde{y}^{3}=\left.1\right|_{\omega} \widetilde{u} & \text { in } \quad \Omega \times(T / 2, T), \\ \widetilde{y}=0 & \text { on } \quad \partial \Omega \times(T / 2, T) \\ \widetilde{y}(\cdot, T / 2)=\widehat{y}(\cdot, T / 2) & \text { in } \quad \Omega\end{cases}
$$

satisfies $\widetilde{y}(\cdot, T)=0$.

Put

$$
y(\cdot, t)= \begin{cases}\widetilde{y}(\cdot, t) & \text { for } t \in(0, T / 2), \\ \widehat{y}(\cdot, t) & \text { for } t \in[T / 2, T],\end{cases}
$$

then $y$ satisfies (1) in case $\gamma=1$ with

$$
u(\cdot, t)= \begin{cases}0 & \text { for } t \in(0, T / 2), \\ \widetilde{u}(\cdot, t) & \text { for } t \in[T / 2, T]\end{cases}
$$

and $y(\cdot, T)=0$. This completes the proof of Corollary 1 .

Acknowledgments. The author would like to express her gratitude to both Referees of this Journal for the valuable comments, important suggestions and corrections of this work which improved substantially the first version of this article.

\section{References}

[1] S. Anita and D. Tataru, Null controllability for the dissipative semilinear heat equation, Appl. Math. Optim. 46 (2002), no.2-3, 97-105. 
[2] T. Cazenave and A. Haraux, An introduction to semilinear evolution equations. Oxford Lecture Series in Mathematics and its Applications, 13. The Clarendon Press, Oxford University Press, New York, 1998.

[3] J-M. Coron, S. Guerrero and L. Rosier, Null controllability of a parabolic system with a cubic coupling term, SIAM J. Control Optim. 48 (2010), no.8, 5629-5652.

[4] J-I. Diaz and J-L. Lions, On the approximate controllability for some explosive parabolic problems. (English summary) Optimal control of partial differential equations (Chemnitz, 1998), 115-132, Internat. Ser. Numer. Math., 133, Birkhäuser, Basel, 1999.

[5] E. Fernández-Cara and S. Guerrero, Global Carleman inequalities for parabolic systems and applications to controllability, SIAM J. Control Optim. 45 (2006), no.4, 1399-1446.

[6] E. Fernández-Cara and E. Zuazua, Null and approximate controllability for weakly blowing up semilinear heat equations, Ann. Inst. H. Poincaré Anal. Non Linéaire 17 (2000), no.5, 583-616.

[7] A. Fursikov and O. Imanuvilov, Controllability of Evolution Equations, Lecture Notes vol. 34 (1996), Seoul National University, Korea.

[8] G. Lebeau and R. Robbiano, Contrôle exact de l'équation de la chaleur, Comm. P.D.E. 20 (1995), no. 1-2, 336-356.

[9] Y. Liu, T. Takahashi and M. Tucsnak, Single input controllability of a simplified fluid-structure interaction model, ESAIM Control Optim. Calc. Var. 19 (2013), no.1, 20-42.

[10] K-D. Phung and G. Wang, An observability estimate for parabolic equations from a measurable set in time and its applications, J. Eur. Math. Soc. 15 (2013), no.2, 681-703.

[11] K-D. Phung, L. Wang and C. Zhang, Bang-bang property for time optimal control of semilinear heat equation, Ann. Inst. H. Poincaré Anal. Non Linéaire 31 (2014), no.3, 477-499.

[12] G. Wang and C. Zhang, Observability inequalities from measurable sets for some evolution equations, preprint arXiv:1406.3422.

[13] E. Zuazua, Controllability and observability of partial differential equations: Some results and open problems, Handbook of Differential equations: evolutionary equations. Vol. III, 527-621, Handb. Differ. Equ., Elsevier/North-Holland, Amsterdam, 2007. 